\title{
Stellar Kinematic Evidence for Massive Black Holes Revisited
}

\author{
HANS-WALTER RIX \\ Institute for Advanced Study, Princeton, NJ, 08540, U.S.A.
}

\begin{abstract}
The existing stellar kinematic evidence for massive black holes at the centers of galaxies is based on $\lesssim 30 \%$ discrepancies between constant M/L models and kinematic data. We show that asymmetries in the observed velocity distributions may introduce systematic errors in the data/model comparisons which are of the same order and have the same sign as these discrepancies. Existing modeling has so far failed to account properly for these asymmetries. Major axis data of M32 are analyzed as a specific example.
\end{abstract}

Key words: galaxies: kinematics - galaxies: dynamics - black holes

After a decade of work (see Kormendy 1992, for a summary) there are by now half a dozen nearby galaxies for which the presence of a central, massive dark object, presumably a black hole (BH), has been inferred, because dynamical models with constant mass-to-light ratio, herafter $(\mathrm{M} / \mathrm{L})_{\text {const }}$, failed to match the observed kinematics. Even though the inferred $\mathrm{BH}$ masses and the distances to the host galaxies vary by more than three orders of magnitude, the gravitational radius, $R_{\text {grav }} . \equiv G M_{B H} /\left\langle\sigma_{\text {galaxy }}^{2}\right\rangle$, which is the distance out to which the BH significantly impacts the stellar kinematics, subtends an angle of $\sim 1^{\prime \prime}$ in every case and is thus always close to the resolution limit (see Figure 1). Leaving all other potential implications aside, this apparent correlation implies that in all cases the discrepancy between the predicted kinematics of a $\mathrm{M} / \mathrm{L}_{c}$ model and the observed kinematics is $\$ 30 \%$ over the observed radial range. Consequently, to reject $(\mathrm{M} / \mathrm{L})_{\text {const }}$. models we must feel confident that we can compare a given dynamical model with the kinematic data to at least that accuracy.

For most of the $\mathrm{BH}$ candidates this data/model comparison is complicated by the fact the observed line-of-sight velocity distribution (LOSVD) is significantly asymmetrical. Such asymmetries may be due to (1) the "intrinsic" distribution function, (2) the line-of-sight integration in the presence of a steep velocity gradient, and (3) seeing. Existing modeling (e.g. Tonry 1987, Kormendy and Richstone 1992) takes effects (2) and (3) into account, but assumes that the velocity distribution is locally a Gaussian.

We have analyzed the LOSVD of M32, NGC 3377 and NGC 3115 (see Rix and White, 1992, for the technique) and have found them to be significantly asymmetrical at small projected radii $\lesssim 1.5^{\prime \prime}$. Specifically, we found for M32 that these asymmetries are much too strong to be attributed mainly to effects (2) and (3) and must reflect a highly anisotropic distribution function.

With such strong asymmetries the $1^{\text {st }}$ and $2^{\text {nd }}$ moment of the LOSVD (the quantities predicted, e.g., by Jeans equation models) differ from the velocity $v$ and the dispersion $\sigma$ estimated from the data by standard means, e.g. cross-correlation. Since existing models for most $\mathrm{BH}$ candidate galaxies predict much weaker asymmetries than are observed, this comparison of moments is necessarily flawed. Figure 2 shows that for M32 the difference between the moments and $v$ and $\sigma$ amounts to $\sim 20 \%$, comparable to the discrepancy between the "conventionally" measured kinematics and the $(\mathrm{M} / \mathrm{L})_{\text {const }}$. models. 


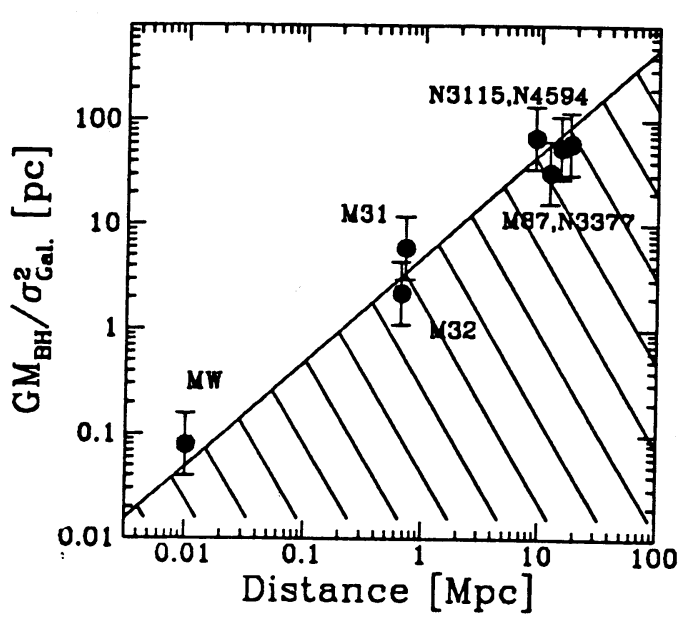

Figure 1

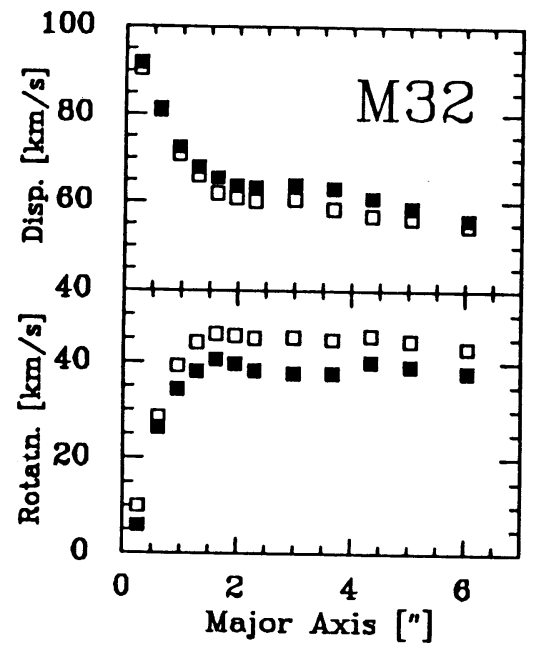

Figure 2

Figure 1: This figure compares the distances of the host galaxies with the gravitational radii of the claimed BH, taken from the literature (e.g. Kormendy 1992, and references therein). The shaded area represent the region of parameter space in which $R_{\text {grav }}$ subtends less than one second of arc. The error bars represent a typically quoted uncertainty of a factor of 3 in the BH mass. In all cases the gravitational radius has an angular size of $\sim 1^{\prime \prime}$ and is thus very close to the observational resolution limit.

Figure 2: This figure compares the the first two moments (filled symbols) of the observed LOSVD with the best fitting Gaussian parameters, $v$ and $\sigma$, in major axis spectra of M32. The LOSVD is sufficiently asymmetrical that the two quantities differ by $\sim 20 \%$.

The existence of strong asymmetries in the LOSVDs of nearly all BH candidates casts doubt on one's ability to compare models with the data at the required accuracy level $(\sim 25 \%)$ with the methods employed so far. At present, it is an open question whether it will be possible to construct $(\mathrm{M} / \mathrm{L})_{\text {const }}$. models, since no selfconsistent models producing sufficient LOSVD asymmetries exist. Nonetheless, it is clear that not all "plausible" (M/L) const. models have been explored and rejected a necessary condition to conclusively infer the presence of unseen mass - and that indeed the models tried so far are incompatible with the observations merely on the basis of their LOSVDs.

The author acknowledges support through Hubble Fellowships from STScI under contract to NASA (HF-1027.01-91A) and is grateful to Dave Carter for permitting the presentation of his data on M32.

\section{References}

Tonry, J. (1987), Ap.J., 332, 932.

Rix, H.- W. and White, S. D. M. (1992), MNRAS, 254, 389.

Kormendy, J. and Richstone, D. (1992), Ap.J., 393, 559.

Kormendy, J. (1992) in High Energy Neutrino Astrophysics, eds. V. Stenger et al., World Scientific 